\title{
Raman spectroscopic evidence for multiferroicity in rare earth nickelate single crystals
}

\author{
I. Ardizzone, ${ }^{1}$ J. Teyssier, ${ }^{1}$ I. Crassee $\odot,{ }^{1}$ A. B. Kuzmenko, ${ }^{1}$ D. G. Mazzone,${ }^{2}$ D. J. Gawryluk $\odot,{ }^{2}$ \\ M. Medarde, ${ }^{2}$ and D. van der Marel $\odot^{1, *}$ \\ ${ }^{1}$ Department of Quantum Matter Physics, University of Geneva, 24 Quai Ernest-Ansermet, 1211 Geneva 4, Switzerland \\ ${ }^{2}$ Paul Scherrer Institut, PSI, Forschungsstrasse 111, 5232 Villigen, Switzerland
}

(Received 19 January 2021; revised 29 March 2021; accepted 18 May 2021; published 2 July 2021)

\begin{abstract}
The rare earth nickelates $\mathrm{RNiO}_{3}$ are metallic at high temperatures and insulating and magnetically ordered at low temperatures. The low temperature phase has been predicted to be type II multiferroic, i.e., ferroelectric and magnetic order are coupled and occur simultaneously. Confirmation of those ideas has been inhibited by the absence of experimental data on single crystals. Here we report on Raman spectroscopic data of $\mathrm{RNiO}_{3}$ single crystals $(\mathrm{R}=\mathrm{Y}, \mathrm{Er}, \mathrm{Ho}, \mathrm{Dy}, \mathrm{Sm}, \mathrm{Nd})$ for temperatures between 10 and $1000 \mathrm{~K}$. Entering the magnetically ordered phase we observe the appearance of a large number of additional vibrational modes, implying a breaking of inversion symmetry expected for multiferroic order.
\end{abstract}

DOI: 10.1103/PhysRevResearch.3.033007

\section{INTRODUCTION}

During recent years materials with the perovskite structure have become the subject of intensive research in the field of photovoltaic cells [1], battery engineering [2], and cancer therapy [3]. In particular, the nickelates with the general formula $\mathrm{RNiO}_{3}$ (with $\mathrm{R}$ a trivalent rare earth) are good candidates for these applications by virtue of the interdependence in these compounds of the structural [4-9], electronic [10-14], and magnetic [15-22] order parameters. The most characteristic property of $\mathrm{RNiO}_{3}$ is the transition from a high-temperature metallic phase to a low-temperature-insulating phase. The temperature of this transition, $T_{M I}$, depends strongly on the radius of the $R^{3+}$ ion: For $\mathrm{R}$ from Lu to $\mathrm{Pr}$, the cell parameter increases almost $10 \%$, while $T_{M I}$ reduces from $600 \mathrm{~K}(\mathrm{Lu})$ to $135 \mathrm{~K}(\mathrm{Pr})$. In the high-temperature metallic phase, the unit cell contains four formula units, where the four nickel atoms occupy equivalent lattice positions corresponding to identical electronic configurations. On entering the insulating phase, a differentiation occurs in the local electronic configuration of the nickel atoms, leading to a modified unit cell containing two pairs of nonequivalent nickel atoms characterized by short and long bonds with the surrounding oxygen atoms [23-27]. This phenomenology is now widely agreed on and has been labeled in the literature as, among other things, "bond disproportionation" and "breathing distortion," the latter of which we employ in this paper. This is accompanied by a slight opening of the angle between the $a$ and $c$ axes away from 90 degrees of the order of 0.1 degree [28] corresponding to

\footnotetext{
*dirk.vandermarel@unige.ch

Published by the American Physical Society under the terms of the Creative Commons Attribution 4.0 International license. Further distribution of this work must maintain attribution to the author(s) and the published article's title, journal citation, and DOI.
}

the monoclinic structure $\left(P 2_{1} / n\right)$, whereas the metallic phase is orthorhombic (Pbmn). For the larger $\mathrm{Nd}$ and Pr ions, the insulating state is magnetically ordered up to $T_{M I}$ [29-32]. For $\mathrm{RNiO}_{3}$ with the smaller rare earth atoms $(\mathrm{R}=\mathrm{Lu}, \mathrm{Y}, \mathrm{Er}, \mathrm{Ho}$, Dy, Sm), the Ni atoms order magnetically at a temperature $T_{N}$ (the Néel temperature) well below $T_{M I}$ and the changes in the lattice parameters at $T_{M I}$ are less pronounced than with $\operatorname{Pr}$ and $\mathrm{Nd}$.

Various different types of magnetic order have been inferred from neutron diffraction, soft $\mathrm{x}$-ray resonant diffraction and resonant inelastic x-ray scattering (RIXS) [15,17,18,33$35]$, all of them characterized by the lack of an inversion center, but differing in the collinear or noncollinear arrangement of the magnetic moments associated to the two types of (short- and long-bond) nickel sites. Theoretically it has been predicted that the combination of breathing distortion and noncentrosymmetric magnetic order can induce a breaking of the inversion symmetry also in the crystal lattice and thus induces an electric dipole, making them type-II multiferroics. [36-45].

\section{RESULTS FOR THE PARAMAGNETIC PHASE}

Small single crystals of $\mathrm{RNiO}_{3}$ with $\mathrm{R}=\mathrm{Y}$, Er, Ho, Dy, Sm, Nd were prepared as described in Ref. [28]. A $\mathrm{DyNiO}_{3}$ crystal is shown in Fig. 1(a). We measured the Raman spectra of $\mathrm{RNiO}_{3}$ crystals with six different compositions in a broad range of temperatures. We monitored the change of the spectroscopic features as the materials pass through three different phases, namely metallic at high temperatures, paramagnetic insulating at intermediate temperatures, and magnetically ordered at the lowest temperatures. The experimental procedures are detailed in Appendix A. The Raman spectra of $\mathrm{DyNiO}_{3}$ are shown in Fig. 1(b) for representative temperatures in the metallic phase $(729 \mathrm{~K})$, deep inside the paramagnetic insulator phase $(205 \mathrm{~K})$, and deep inside the magnetically ordered phase (12 K). In Fig. 1(c) 
(a)

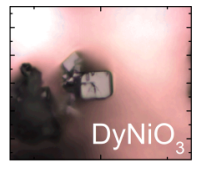

$\longmapsto 20 \mu \mathrm{m}$

(c) $\quad \log _{10}$ (Intensity) $-0.9 \square 0.3$
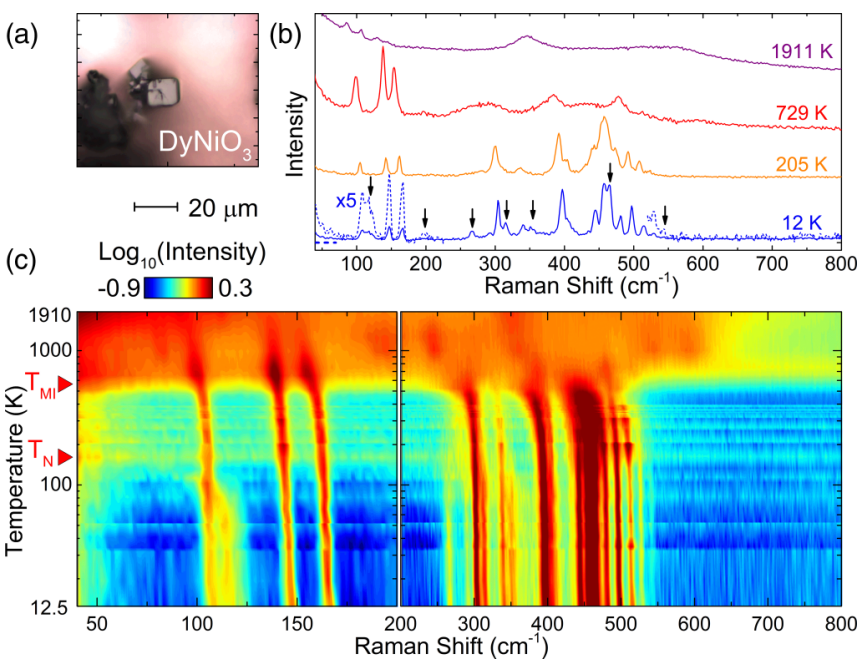

FIG. 1. (a) Image of a $\mathrm{DyNiO}_{3}$ single crystal inside the cryostat. (b) Typical Raman spectra recorded on $\mathrm{DyNiO}_{3}$ at temperatures in the antiferromagnetic-insulating phase (blue curve, dashed line is a factor 5 magnification of the blue curve), the paramagnetic-insulating phase (orange curve), and the metallic paramagnetic phase (red curve). The purple curve is close to the melting point of the material. The curves have been given vertical offsets to avoid clutter. Raman modes appearing in the antiferromagnetic phase are marked by arrows. (c) Colormap of the Raman response in the full temperature range from 12.5 to $1911 \mathrm{~K}$. Metal-insulator and magnetic transitions are labeled as red symbols. the full data set of $\mathrm{DyNiO}_{3}$ covering the entire temperature range is represented as a colormap of the Raman response in the frequency-temperature plane (see Appendix A for the other samples). For all samples the evolution of the phonon frequencies as a function of temperature is summarized in Fig. 2. We begin with an observation on the character of the Raman modes. In the metallic and insulating phases, the crystal point group has, at least down to $T_{N}$, inversion symmetry. Consequently, the optical modes are either Raman active (even parity) or dipole active (odd parity). The Pbmn (metallic phase at high temperature) and the $P 2_{1} / n$ (insulating phase at low temperature) space groups contain four formula units and therefore have 60 vibrational degrees of freedom corresponding to 3 acoustic modes, 33 odd parity modes observable in the optical conductivity, and 24 even parity modes that are Raman active. Another important feature shared by the $P 2_{1} / n$ and Pbmn phases is that the four Ni atoms are at centers of inversion symmetry. Consequently only the motions of oxygens and rare earth atoms contribute to the Raman modes.

The overall trend of the mode frequencies as a function of rare earth element (Fig. 2) is a blueshift of all frequencies when moving from $\mathrm{Nd}$ to $\mathrm{Y}$. This trend is most pronounced for the three main modes below $230 \mathrm{~cm}^{-1}$, where in particular the case of $\mathrm{YNiO}_{3}$ stands out in that the three main modes have frequencies approximately $35-40 \%$ higher than for $\mathrm{HoNiO}_{3}$, in agreement with the ratio $\left(m_{H o} / m_{Y}\right)^{0.5}=1.36$ expected for vibrations with a rare earth character mainly. The modes above $500 \mathrm{~cm}^{-1}$ shift up by approximately $10 \%$ from

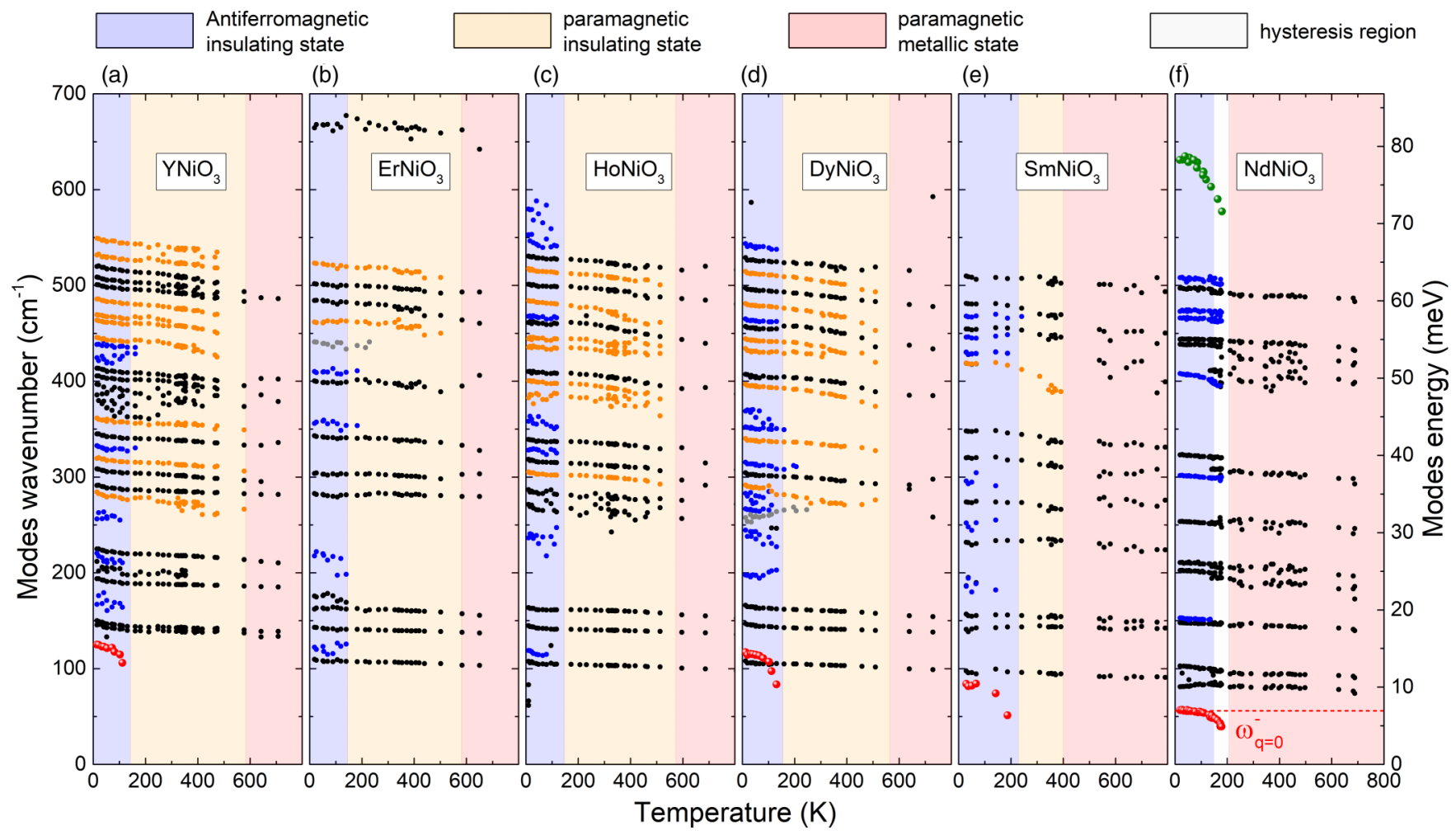

FIG. 2. Evolution with temperature of the frequency of the Raman modes. Black symbols: Modes already present above $T_{M I}$. Orange symbols: Additional modes below $T_{M I}$. Blue symbols: Additional modes below $T_{N}$. Red symbols: Antiferromagnetic resonance. Gray symbols for $\mathrm{ErNiO}_{3}$ and $\mathrm{DyNiO}_{3}$ : Additional modes appearing halfway $T_{M I}$ and $T_{N}$. Green symbols $\left(\mathrm{NdNiO}_{3}\right)$ : Bimagnon bound state (tentative; see Discussion). 
Nd to Y, revealing a hardening of the chemical bond, a trend that is expected in view of the reduction of the lattice constant for the smaller rare earth elements. Cooling through $T_{M I}$ the four $\mathrm{NiO}_{6}$ octahedra within the primitive cell bifurcate into one set with short Ni-O bonds and a second set with long bonds. While the number of Raman-active modes (24) is not affected by the symmetry breaking, we may reasonably expect that intensity and frequency of these modes change at the transition. For all $\mathrm{RNiO}_{3}$ compounds of this study, the metalinsulator phase transition results in a significant blueshift of all modes (Figs. 1 and 2). Above $T_{M I}$ the vibrational features are much broader than below $T_{M I}$. Consequently a number of modes that are close in frequency can be separated from each other below $T_{M I}$ (marked in orange in Fig. 2) but not above $T_{M I}$. A weak mode at $450 \mathrm{~cm}^{-1}$ for $\mathrm{ErNiO}_{3}$ and one at $260 \mathrm{~cm}^{-1}$ for $\mathrm{DyNiO}_{3}$ broaden progressively as a function of increasing temperature (gray symbols in Fig. 2) and become indistinguishable midway $T_{N}$ and $T_{M I}$. The fact that they show up far above $T_{N}$ indicates that these are Raman-active modes of the paramagnetic phase.

\section{RESULTS FOR THE ANTIFERROMAGNETIC PHASE}

Cooling the samples through the magnetic phase transition has a surprisingly large impact on the Raman spectra, in particular showing a large number of additional modes (see Fig. 2) which we interpret as phonons, with a few exceptions that we will discuss first: For all compounds except $\mathrm{ErNiO}_{3}$ and $\mathrm{HoNiO}_{3}$, we observe an additional mode at the low-frequency end of the spectrum, showing a strong redshift on raising the temperature to $T_{N}$. For $\mathrm{NdNiO}_{3}$ the frequency is $58 \mathrm{~cm}^{-1}$ at the lowest temperatures. Since this is in the range of the zone-center magnon obtained for the same material from extrapolating RIXS data $(13 \mathrm{meV})$ [35], we interpret this mode as the antiferromagnetic resonance. $\mathrm{NdNiO}_{3}$ is the only compound showing a peak near $640 \mathrm{~cm}^{-1}$ with a strong redshift on raising the temperature to $T_{N}$. This is probably a bimagnon: Taking the experimental value of the energy of the zone-boundary magnon from RIXS [35] we arrive at $2 \times 55 \mathrm{meV}$ giving $887 \mathrm{~cm}^{-1}$ as the upper bound. The lower frequency in the Raman spectrum can be qualitatively understood as a consequence of magnonmagnon coupling.

As is illustrated in Fig. 1(b) for the case of $\mathrm{DyNiO}_{3}$, we observe below $T_{N}$ at least five relatively strong additional modes at $120,270,310,350$, and $460 \mathrm{~cm}^{-1}$ and two weaker ones at 200 and $540 \mathrm{~cm}^{-1}$. The total number of modes in the low-temperature Raman spectrum is 22 out of 24 that are Raman active, i.e., the number of Raman-active modes observed in $\mathrm{DyNiO}_{3}$ does not indicate as such that the space group in the magnetic phase should be different from $P 2_{1} / n$. However, it is remarkable that certain modes appear in pairs. For $\mathrm{DyNiO}_{3}$ the modes near 305, 340, and $450 \mathrm{~cm}^{-1}$ split into doublets below $T_{N}$. The splitting of the $340 \mathrm{~cm}^{-1}$ mode is also clearly seen for $\mathrm{HoNiO}_{3}$ and $\mathrm{ErNiO}_{3}$ and for some of the other modes in specific samples. $\mathrm{NdNiO}_{3}$ is special among the samples studied in that we see for all phonons an abrupt narrowing and an intensity change at $T_{N}\left(=T_{M I}\right)$, and the $305 \mathrm{~cm}^{-1}$ mode splits into a strong peak at $300 \mathrm{~cm}^{-1}$ and a weak one at $315 \mathrm{~cm}^{-1}$, confirming the observations in Refs. [46,47]. For $\mathrm{DyNiO}_{3}$, a well-defined mode appears near

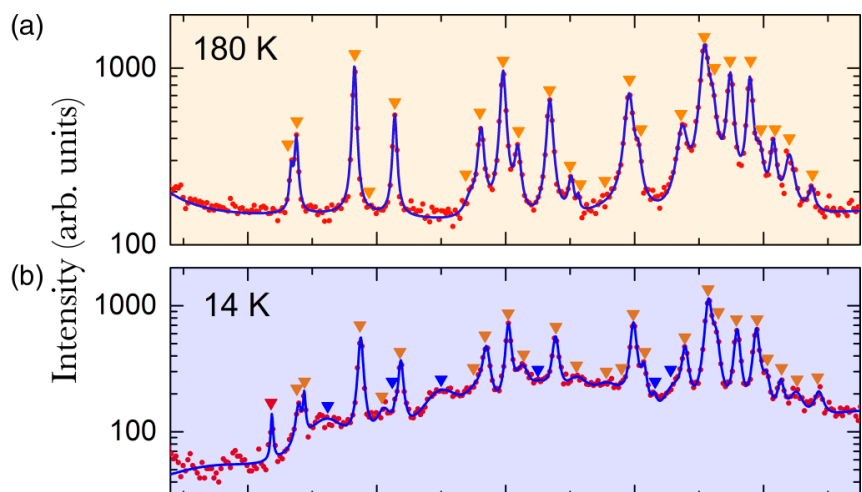

(c)

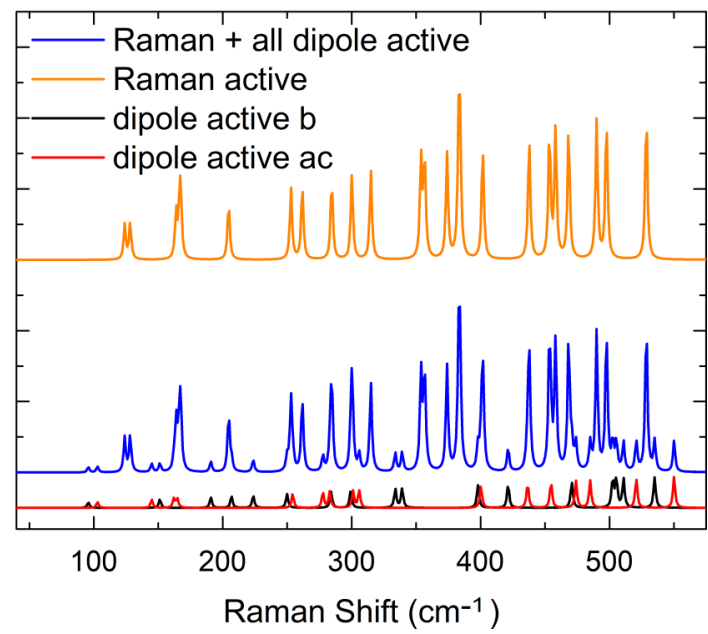

FIG. 3. Raman spectra of an $\mathrm{YNiO}_{3}$ single crystal (a) in the paramagnetic-insulating phase $(180 \mathrm{~K})$ and $(\mathrm{b})$ in the antiferromagnetic-insulating phase (14 K). Orange triangles: Raman modes of the paramagnetic phase. Blue triangles: Additional modes in the magnetic phase. Red: Antiferromagnetic resonance. (c) Simulated Raman spectrum of $\mathrm{YNiO}_{3}$ in the paramagnetic-insulating phase (orange) and the insulating antiferromagnetic phase (blue), where it is assumed that all dipole-active phonons ( $b$ axis, black; $a c$ plane, red) have become Raman active as a result of inversion symmetry breaking. The calculation of the phonon frequencies is described in Appendix B. The modes have a Lorentz line-shape with a full width at half-maximum of $4 \mathrm{~cm}^{-1}$. The amplitudes $f_{j}$ of the Raman modes [see Eq. (A3)] were all given the same value and the amplitudes of all polar modes $1 / 5$ of those of the Raman modes.

$280 \mathrm{~cm}^{-1}$ surrounded by two shoulders. The same mode is observable at low temperature for $\mathrm{HoNiO}_{3}$ with the difference that this narrow peak is still present as a shoulder in the paramagnetic phase. We observe also the appearance of a broad mode at $250 \mathrm{~cm}^{-1}$, visible for $\mathrm{YNiO}_{3}, \mathrm{HoNiO}_{3}$, and $\mathrm{DyNiO}_{3}$ (Fig. 2). A second well-defined characteristic mode appears at around $350 \mathrm{~cm}^{-1}$ for $\mathrm{HoNiO}_{3}, \mathrm{DyNiO}_{3}$, and $\mathrm{ErNiO}_{3}$. Other new modes emerge at 550 and $570 \mathrm{~cm}^{-1}$ for $\mathrm{HoNiO}_{3}$, at 200 and $550 \mathrm{~cm}^{-1}$ for $\mathrm{DyNiO}_{3}$ and at $440 \mathrm{~cm}^{-1}$ for $\mathrm{ErNiO}_{3}$.

For the interpretation of the additional modes below $T_{N}$, the case of $\mathrm{YNiO}_{3}$ is of particular importance. Taking a close look at the Raman spectra in the paramagnetic and antiferromagnetic-insulating phase shown in Figs. 3(a) and 3(b), we notice that (i) there are 24 Raman modes above $T_{N}$ and (ii) this number increases to 31 below $T_{N}$. One of the 
(a) T-type

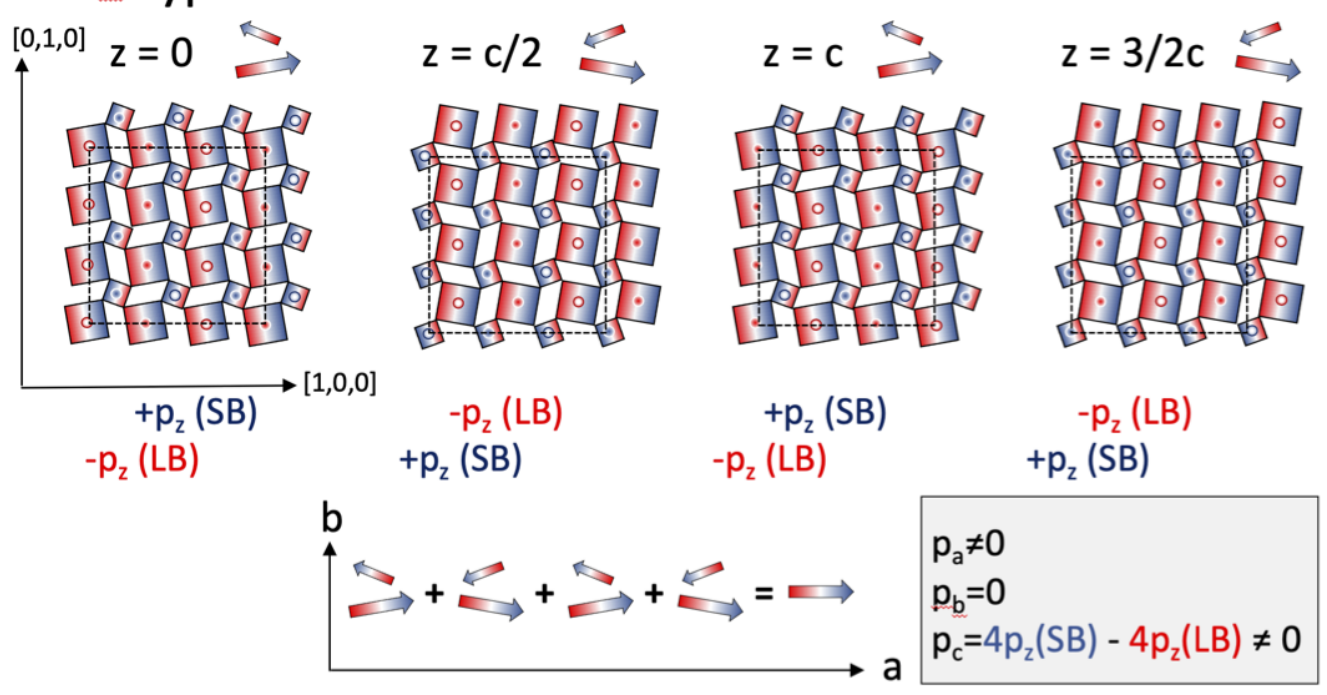

(b) S-type

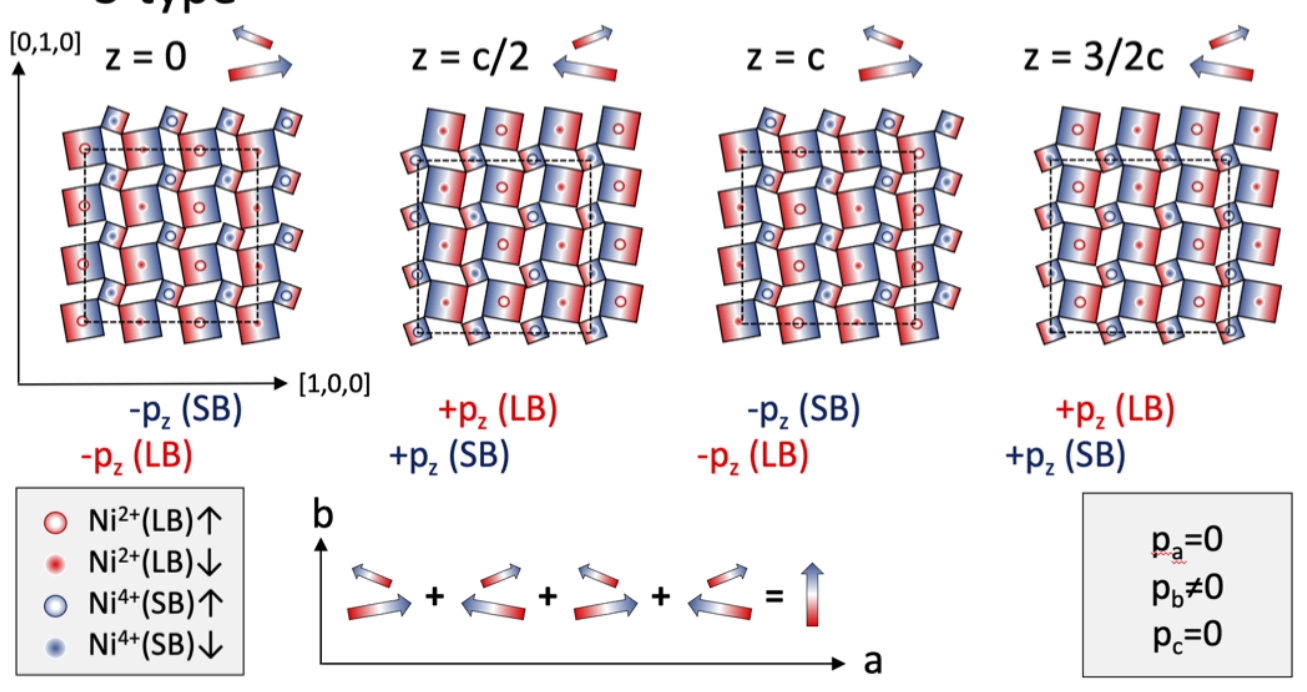

FIG. 4. Multiferroicity of (a) $T$-type and (b) $S$-type antiferromagnetic order [39]. Coordinate axes are labeled according to $P 2_{1} / n$ setting. Dotted squares indicate the alignment of the planes stacked along $[0,0,1]$. Labels for up/down spin and long-/short-bond $\mathrm{Ni}$ are given in the top right.

extra modes (the one at $120 \mathrm{~cm}^{-1}$ ) is the antiferromagnetic resonance. Since $Y$ has no $4 f$ electrons, we can exclude beyond any doubt contributions of $4 f$ crystal field excitations to the Raman spectra. The only remaining interpretation of the additional modes is therefore that these are phonons. Since for $P 2_{1} / n$ the number of Raman-active phonons is limited to 24 , observing 30 Raman-active phonons is a clear demonstration that the space group in the antiferromagnetic phase must be different from $P 2_{1} / n$.

\section{DISCUSSION}

It is fully established that the magnetic order of these compounds is described by the propagation vector $(1 / 2,0,1 / 2)$ \{ or $(1 / 4,1 / 4,1 / 4)$ when referred to the underlying pseudocubic lattice [48]\}, but the orientation of the magnetic moments is less clear [15,17,18,33-35]. Giovannetti et al. [39] discuss three types of magnetic order labeled $T, S$, and $N$. For $T$-type order, the magnetic moments are arranged in planes of parallel spins alternating as $\uparrow \uparrow \downarrow \downarrow$ along the $[1,1,1]$ direction of the underlying pseudocubic structure, which they predict to be polar with electric dipole components along $a$ and $c$. For $S$ type, they predict a net electric dipole along $b$. The predicted multiferroicity for $T$ and $S$ originates in the phenomenon that theoretically regions between parallel (opposite) magnetic moments become electron poor (rich) due to exchange correlation. As illustrated in Fig. 4 this would result in the appearance of a net electric moment, whose direction can be along the $b$ axis or perpendicular to it depending on the type of magnetic order. The noncollinear magnetic structure $N$ owes its multiferroicity to spin-orbit coupling and has an electric dipolar moment much weaker than the collinear $T$ and $S$ arrangements [39]. Perez-Mato et al. predicted the existence of magnetism-induced ferroelectricity from group theory arguments based in the symmetry of the paramagnetic space group and the magnetic propagation vector [43]. According to these 


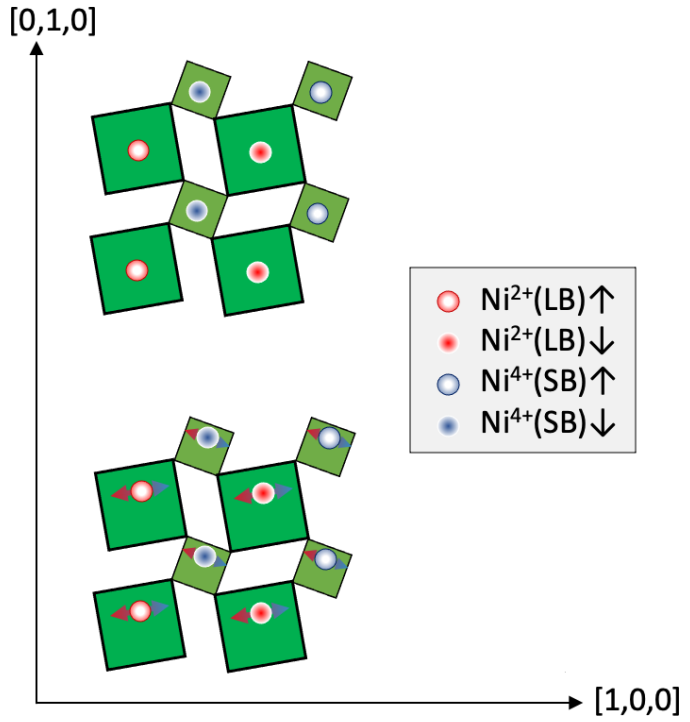

FIG. 5. Scheme of the spontaneous inversion symmetry breaking. Labels for up/down spin and long-/short-bond $\mathrm{Ni}$ are indicated at the right-hand side. To avoid clutter, oxygen atoms (at the corners of the squares) and rare earth atoms (in the open spaces) are not indicated. Top: The Ni ions occupy inversion-symmetric positions. Bottom: The electric fields induced by the T-type or S-type antiferromagnetic order (see Fig. 4) push the Ni ions to low-symmetry positions, which has two main effects: (i) It contributes a net electric dipole moment. (ii) Since these positions lack inversion symmetry, modes of any polarization are simultaneously Raman active and dipole active. Vibrations around the rest position are symbolized by double-headed arrows.

arguments a symmetry decrease from centrosymmetric $P 2_{1} / n$ to polar $P 2_{1}$ and the appearance of spontaneous polarization along the $b$ crystal axis are expected to occur below $T_{N}$ for the collinear and noncollinear magnetic structures reported, respectively, for $\mathrm{PrNiO}_{3}$ [15] and $\mathrm{HoNiO}_{3}$ [17]. In any of these scenarios the electric fields induced by the electronic charge displacements will act as a force on the ions, deforming the lattice and-most significantly-pushing the $\mathrm{Ni}$ ions away from centrosymmetric positions. As illustrated in Fig. 5, this relaxes the optical selection rules. In particular it causes dipole-active vibrations to become simultaneously Raman active and vice versa.

\section{KEY IMPLICATIONS OF THE EXPERIMENTS}

Polarized neutron diffraction experiments have proven the magnetic origin of the aforementioned $(1 / 2,0,1 / 2)$ superlattice [15-17]. Consequently, the magnetic order modulates the force constants with the same lattice periodicity as the one of the paramagnetic state, thus excluding the possibility that the additional modes are folded zone-boundary phonons. If, on the other hand, a breaking of inversion symmetry were to occur, this would have a major impact on the Raman spectra. The implication for the vibrational modes is that all dipole-active modes become simultaneously Raman active and vice versa. For nonpolarized Raman, as used for the spectra in Fig. 3, at most 33 polar modes will then contribute to the Raman spectra below $T_{N}$. The ab initio calculations (see Appendix B) of the
TABLE I. Néel temperature [38], insulator-metal transition temperature [38], rare earth radius (eightfold coordination) [49], and tolerance factor [38] of the $\mathrm{RNiO}_{3}$ compounds that are subject of the present study.

\begin{tabular}{|c|c|c|c|c|}
\hline Compound & $\begin{array}{l}T_{N} \\
\mathrm{~K}\end{array}$ & $\begin{array}{c}T_{M I} \\
\mathrm{~K}\end{array}$ & $r_{R E}$ & $t$ \\
\hline $\mathrm{YNiO}_{3}$ & 143 & 584 & 1.019 & 0.862 \\
\hline $\mathrm{ErNiO}_{3}$ & 145 & 582 & 1.004 & 0.864 \\
\hline $\mathrm{HoNiO}_{3}$ & 147 & 573 & 1.015 & 0.866 \\
\hline $\mathrm{DyNiO}_{3}$ & 155 & 564 & 1.027 & 0.868 \\
\hline $\mathrm{SmNiO}_{3}$ & 229 & 401 & 1.079 & 0.894 \\
\hline $\mathrm{NdNiO}_{3}$ & 203 & 202 & 1.109 & 0.915 \\
\hline
\end{tabular}

vibrational spectrum presented in Fig. 3(c) demonstrate that the features in the experimental data of Fig. 3(b) are indeed consistent with polar modes that become Raman active below $T_{N}$. Some of the additional modes below $T_{N}$ show up as shoulders of peaks already present in the paramagnetic-insulating phase. That some of these modes appear as pairs and others not is then a simple consequence of the fact that some of the Raman and polar modes correspond to similar vibrational patterns, the main difference being the in-phase and out-of-phase motion of subsets of the atoms (in the present case four formula units) inside the primitive cell. Furthermore, we observe a total of 30 vibrational modes at low temperature for $\mathrm{YNiO}_{3}$ which is 6 more than allowed by the factor group analysis. The implication from these results is that the antiferromagnetic phase is polar.

Moving from $\mathrm{Nd}$ to $\mathrm{Y}$, the number of additional modes that we can detect below $T_{N}$ increases. That the multiferroic effect is strongest for the smaller rare earth ions has in fact been anticipated by Giovannetti et al. [39]. The ingredients responsible for the multiferroic effects are magnetic order, breathing distortion, and rotation of the oxygen octahedra (Fig. 4). This combination of factors is uncommon in the multiferroic family tree [45] and characteristic for the rare earth nickelates. Of the samples studied here $\mathrm{YNiO}_{3}$ has the smallest tolerance factor (Table I) and consequently the largest octahedral rotation, highest $T_{M I}$, and the strongest breathing distortion. While the moderate decrease of $T_{N}$ from $\mathrm{NdNiO}_{3}$ to $\mathrm{YNiO}_{3}$ suggests a weakening of the magnetic order parameter, this is more than compensated by the increasing octahedral rotation and breathing distortion, together resulting in the observed positive trend of the multiferroic coupling from large to small tolerance factor.

We see that the Raman spectra provide experimental support for the predicted existence of magnetism-driven ferroelectricity, demonstrating the clear symmetry breaking below $T_{N}$ and confirming previous indirect experimental evidence supporting this idea. For $\mathrm{PrNiO}_{3}$, some of us analyzed the temperature dependence of the crystal structure in terms of symmetry-adapted modes [8]. The unconventional temperature dependence of the lattice parameters below $T_{M I}=T_{N}$, and the observation of a nearly perfect linear correlation between the breathing mode amplitude and the staggered magnetization, lead to the conjecture of a hidden order in the insulating phase, in particular the predicted polar distortion 
induced by the noncentrosymmetric magnetic order $[39,43]$. The clear lattice anomalies recently observed at $\mathrm{T}_{N}$ for $\mathrm{EuNiO}_{3}[50]$ confirm this intuition, giving additional support to the Raman findings. In summary, it has been anticipated theoretically that magnetic order in $\mathrm{RNiO}_{3}$ breaks inversion symmetry, corresponding to a multiferroic state in which all dipole-active phonons are simultaneously Raman active. Our observation of additional Raman-active modes below $T_{N}$ provides experimental for this prediction. Full characterization of the phase below $T_{N}$ requires additional experimental investigations such as second harmonic generation, measurements of the electric dipole field and crystallographic experiments.

The data sets generated and analyzed during the current study are available in Ref. [51] and will be preserved for 10 years.

\section{ACKNOWLEDGMENTS}

This project was supported by the Swiss National Science Foundation through projects 200020-179157, 200021163103, 200020-185061, NCCR MARVEL (Grant No. 51NF40-182892), and Réquip Grant No. 206021-139082.

\section{APPENDIX A: EXPERIMENTS}

The microcrystals [28] were glued on the copper plate of a He flow cryostat (Konti Micro from CryoVac GMBH) using high vacuum grease. Raman spectra were recorded using a Horiba LabRAM HR Evolution spectrometer with an excitation wavelength of $532 \mathrm{~nm}$ and the resolution of $2 \mathrm{~cm}^{-1}$ over the full range. The laser light was focused on a $2-\mu \mathrm{m}$ spot using a window-corrected $63 \times$ objective. Size, shape, and orientation of the incident polarization (parallel to the horizontal axis) varied from one sample to another. Images of some of the single crystals mounted in the cryostat are displayed in Fig. 6, together with the corresponding Raman spectra in the high-temperature metallic phase (red curves) and in the low-temperature antiferromagnetic phase (blue curves). The small size of the crystals prohibited reproducible alignment and concomitant polarization analysis of the Raman spectra.

Spectra were measured in a broad temperature range from 10 to $1000 \mathrm{~K}$ for most samples and for $\mathrm{NdNiO}_{3}$ up to $1911 \mathrm{~K}$. The variable power of the excitation laser was used for heating the microcrystals. The temperature of the sample is determined by the equilibrium of heat exchange between the crystal and the sample holder and heating by different power of the laser. Due to differences in, among others, absorption coefficient, size, shape, and the contact between grease layer, crystal, and copper, the thermal coupling of the crystals to the cold plate varies from one specimen to another. For these reasons the same incident laser power heats up the different crystals to different temperatures. The sample temperature was calculated from the Stokes/anti-Stokes ratio as detailed below. As the laser power had to be varied in order to tune the sample temperature, it was necessary to normalize the spectra presented in Figs. 1 and 7. For the normalization, first, the average intensity of the Stokes response was extracted between 250 and $550 \mathrm{~cm}^{-1}$ and each spectrum was normalized to that average intensity. In a second step, we corrected for the drift between subsequent temperatures. To that end, for
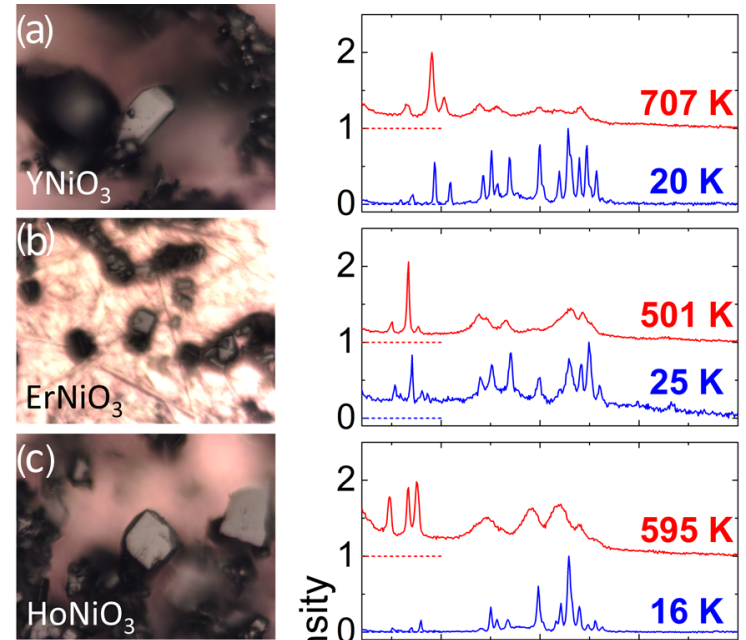

(d)
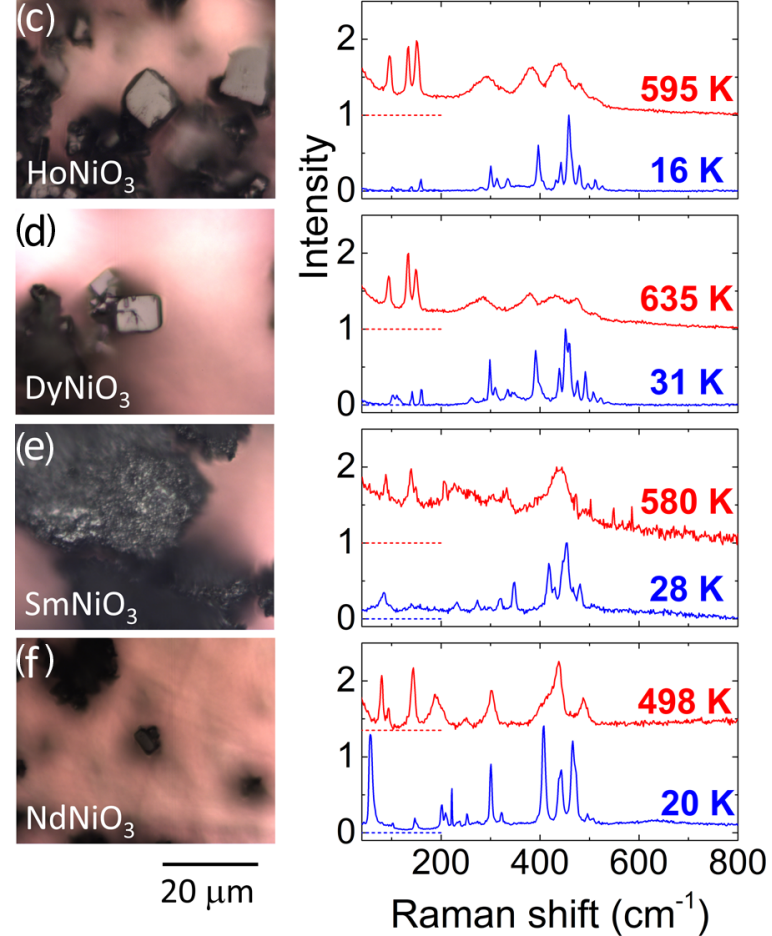

FIG. 6. Images of (a) $\mathrm{YNiO}_{3}$, (b) $\mathrm{ErNiO}_{3}$, (c) $\mathrm{HoNiO}_{3}$, (d) $\mathrm{DyNiO}_{3}$, (e) $\mathrm{SmNiO}_{3}$, and (f) $\mathrm{NdNiO}_{3}$ single crystals inside the cryostat and the associated raw Raman spectra in the hightemperature metallic state (red curve) and in the low-temperature antiferromagnetic state (blue curve). Temperature of each spectrum is indicated on the curve. Scale bar is identical for all samples.

each temperature we extracted the average Stokes response in a frequency range without Raman-active modes and as a function of temperature fitted these averages using a polynomial. Then, for each temperature, the difference between the extracted average and the fitted value was subtracted from the entire Raman spectrum, effectively removing background jitter as a function of temperature.

The Raman spectrum at finite temperatures is given by

$$
I(\omega, T)=\frac{\operatorname{Im} \chi(\omega)}{1-e^{-\hbar \omega / k_{B} T}},
$$

where $\omega=\omega_{\text {in }}-\omega_{\text {out }}$ is the Raman frequency shift, $\omega_{\text {in }}\left(\omega_{\text {out }}\right)$ is the frequency of the incoming (scattered) photon, $T$ is the temperature, $k_{B}$ is the Boltzmann constant, and $\chi(\omega)$ is the Raman susceptibility. The imaginary (real) part of $\chi(\omega)$ is an odd (even) function of $\omega$. Since the denominator of Eq. (A1) changes sign at the origin of $\omega$, the function $I(\omega, T)$ is positive 


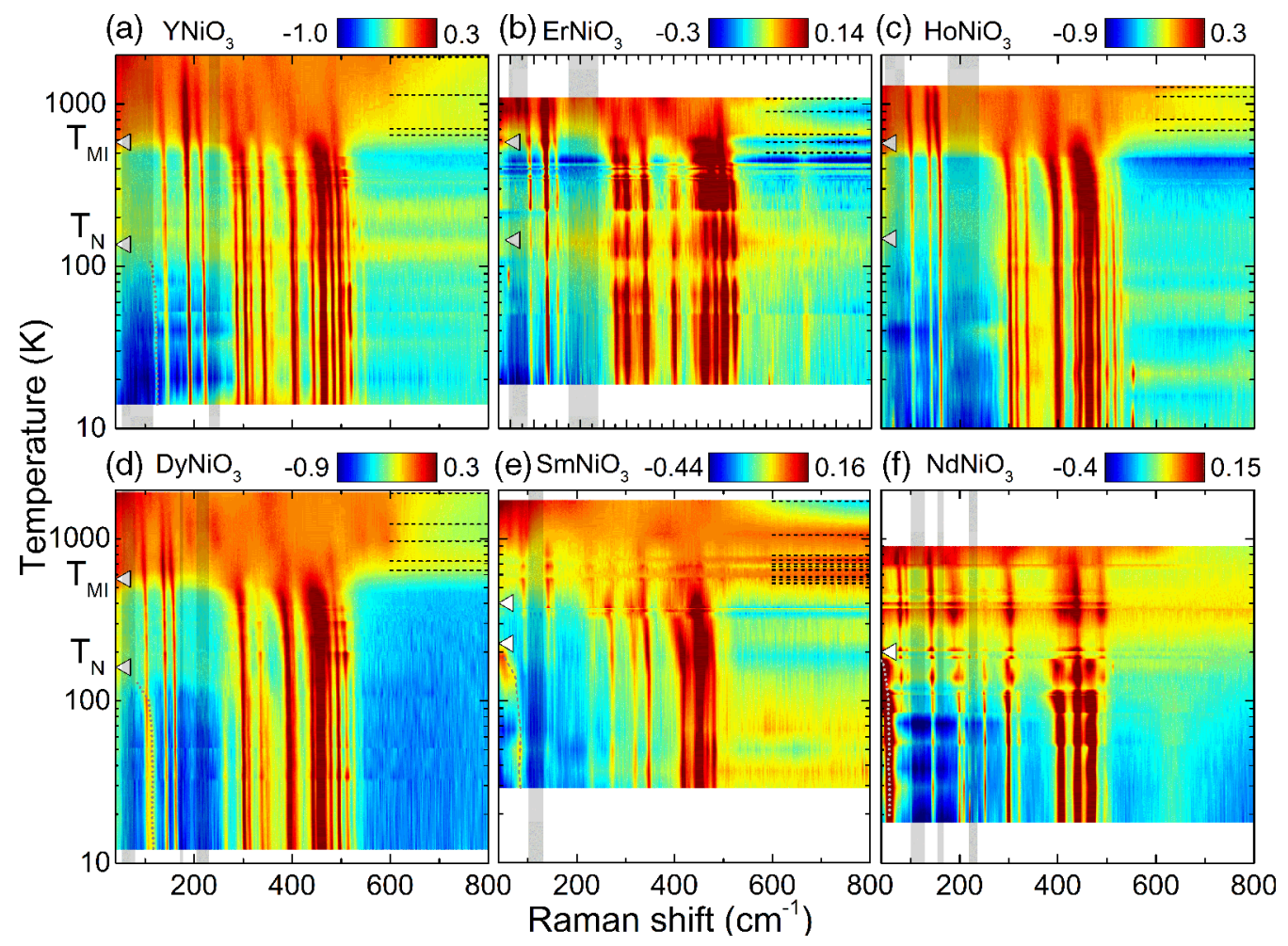

FIG. 7. Colormap of Raman intensity for (a) $\mathrm{YNiO}_{3}$, (b) $\mathrm{ErNiO}_{3}$, (c) $\mathrm{HoNiO}_{3}$, (d) $\mathrm{DyNiO}_{3}$, (e) $\mathrm{SmNiO}_{3}$, and (f) $\mathrm{NdNiO}_{3}$ samples. Horizontal axis is the Raman shift in $\mathrm{cm}^{-1}$, vertical axis is the temperature in $\mathrm{K}$ on a log scale, and color defined the intensity of the signal in a logarithmic scale. White triangles indicate the temperatures of metal to insulator (high temperature) and magnetic ordering (low temperature). When present, the soft-mode frequency from the spectral fit is marked with a dashed gray line. Gray regions are averaging windows used for the temperature dependence of the Raman electronic background. Dotted horizontal lines indicate the temperatures of the spectra above $700 \mathrm{~K}$ used for the colorplot.The temperature mesh below $700 \mathrm{~K}$ is too dense to indicate this way.

for positive and negative $\omega$. Positive (negative) frequencies represent the Stokes (anti-Stokes) side of the spectrum. Since the Stokes/anti-Stokes ratio

$$
\frac{I(\omega, T)}{I(-\omega, T)}=e^{\hbar \omega / k_{B} T}
$$

is a universal function of $\omega / T$, the temperature can in principle be readily obtained from this ratio. In practice one first needs to correct the spectra for the zero-loss peak and for a dark current background. In addition, it is necessary to correct for spectrometer drift of the zero of the frequency axis. Since Eq. (A2) can only be applied after these corrections have been made, we use a different approach, which consists of fitting [52] the spectra for positive and negative Raman shifts to a sum of oscillators $\chi_{j}(\omega)$ for which we use

$$
\chi(\omega)=\sum_{j} \frac{\omega^{2} f_{j}}{\omega_{j}^{2}-\omega\left(\omega+i \gamma_{j}\right)},
$$

where $f_{j}$ parametrizes the amplitude of the $j$ th Raman mode, $\omega_{j}$ its peak frequency, and $\gamma_{j}$ its half-width at half maximum. For all samples, the spectra at different temperatures could be satisfactory modeled with a Lorentzian line shape for the oscillators. The temperature of the sample is used as fitting parameter together with the parameters $\omega_{j}, \gamma_{j}$, and $f_{j}$ of the oscillators. Additional adjustable parameters were the zero of the frequency axis, a frequency-independent background, and a symmetric profile for the zero-frequency peak. We excluded the frequency range $-50 \mathrm{~cm}^{-1}<\omega<50 \mathrm{~cm}^{-1}$ from the fitting procedure for all crystals except $\mathrm{NdNiO}_{3}$ for which the excluded range was $-20 \mathrm{~cm}^{-1}<\omega<20 \mathrm{~cm}^{-1}$. Crucially, the Stokes and anti-Stokes sides of the spectrum were acquired simultaneously and the spectrum for $\omega<0$ and $\omega>0$ was fitted with a single set of parameters. In Fig. 8 the example of $\mathrm{DyNiO}_{3}$ is shown. The sample holder was kept at $20 \mathrm{~K}$. The relative intensity of the Stokes and anti-Stokes peaks reveals that the temperature at the location of the laser beam is $64 \mathrm{~K}$.

\section{APPENDIX B: PHONON CALCULATIONS}

The phonon frequencies presented in Fig. 3(c) were calculated using density functional. Perturbation theory was as implemented in Quantum Espresso [53]. The monoclinic unit cell was relaxed and an all-electrons self-consistent calculation was used with a generalized gradient approximation within the Perdew-Burke-Ernzerhof functional [54]. Cut-off energies for kinetic energy and charge density were 100 and $600 \mathrm{Ry}$, respectively. The $k$ points corresponding to the electronic wave functions were integrated on a $7 \times 6 \times$ 5 Monkhorst-Pack grid yielding $72 k$ points in the irreducible wedge of the Brillouin zone. Dynamical matrices were then computed using a self-consistent convergence criterion of $10^{-12} \mathrm{Ry}$. The structure was relaxed to self-consistent 


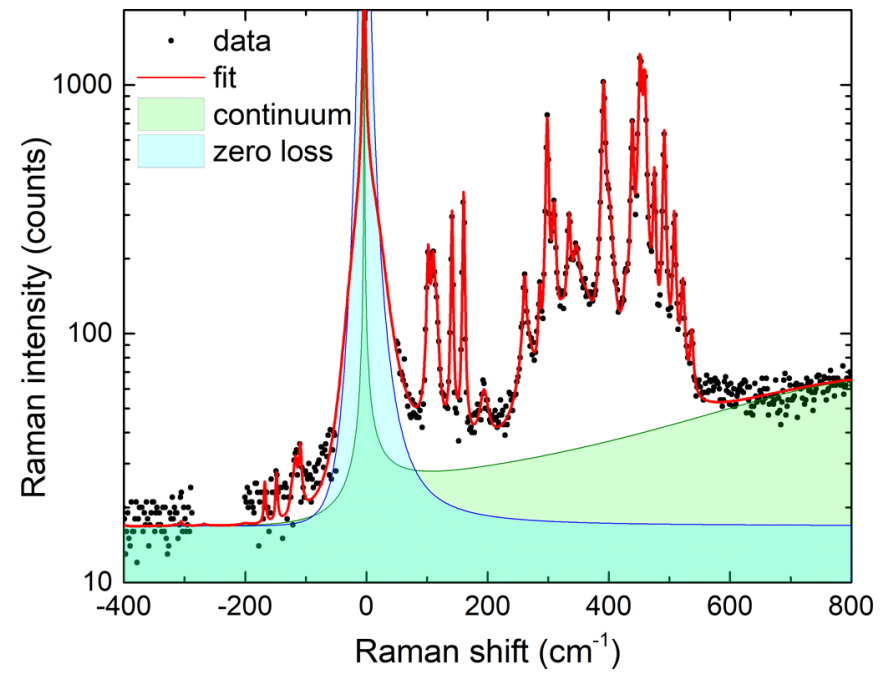

FIG. 8. Raman spectrum of $\mathrm{DyNiO}_{3}$ shown together with a multioscillator fit obtained by adjusting the temperature, the oscillator parameters of Eq. (A3), a background, and the zero of frequency.

convergency, with the effect that lattice parameters do not exactly match the experimental values.

\section{APPENDIX C: MAGNETIC SPACE GROUP AND MULTIFERROICITY}

The magnetic propagation vector of the $\mathrm{RNi} O_{3}$ expressed on the reciprocal lattice basis of the $P 2_{1} / n$ space group is
$(1 / 2,0,1 / 2) . P 2_{1} / c$ describes the same space group and has the same $\vec{a}$ and $\vec{b}$ primitive lattice vectors, but $\vec{c}_{c}$ of $P 2_{1} / c$ equals $\vec{a}+\vec{c}_{n}$ of $P 2_{1} / n$. Correspondingly, the magnetic propagation vector expressed on the reciprocal lattice basis of $P 2_{1} / c$ is $(1 / 2,0,0)$, i.e., the $P 2_{1} / c$ setting reveals that this magnetic order doubles the volume of the nonmagnetic primitive cell. In Hermann-Mauguin notation, the symbol $2{ }_{1}$ refers to a twofold screw axis parallel to the unique $(b)$ axis of the monoclinic structure and the symbol $/ c$ to a glide plane, more specifically, a mirror operation in a plane perpendicular to $b$ followed by a translation along $\vec{c}_{c} / 2[55,56]$. If the glide plane symmetry is broken, the corresponding magnetic space group (MSG) $P_{2 a} 2_{1}$ supports a net electric dipole along $\vec{b}$ [56]. Likewise, for MSG $P_{2 a} c$ the screw axis symmetry is broken, resulting in a net electric dipole with components along $\vec{a}$ and $\vec{c}_{c}$ [56]. $S$-type and $T$-type order belong to MSG $P_{2 a} 2_{1}$ and $P_{2 a} c$, respectively. For the microscopic description of multiferroicity in $T$ - and $S$-type order, we follow Giovannetti et al. [39] and use $P 2_{1} / n$ setting; correspondingly from hereon, $c$ refers to $c_{n}$. Long-bond (LB) and short-bond (SB) Ni sites alternate along $\vec{a}+\vec{b}, \vec{a}-\vec{b}$ and $\vec{c}$, with an octahedral counter-rotation in the $a b$ plane of the two types of sites. Exchange correlation causes electron depletion (accumulation) between $\mathrm{Ni}$ neighbors of parallel (opposite) spin. In the case of T-type order [Fig. 4(a)], the net dipole of the planes together is zero along $\vec{b}$ and finite along $\vec{a}$ and $\vec{c}$. In the case of S-type order [Fig. 4(b)], the net dipole is zero along $\vec{a}$ and $\vec{c}$, and finite along $\vec{b}$.
[1] C. Kalaiselvi, N. Muthukumarasamy, D. Velauthapillai, M. Kang, and T. Senthil, Importance of Halide Perovskites for next generation solar cells-A review, Mater. Lett. 219, 198 (2018).

[2] H. Xu, P.-H. Chien, J. Shi, Y. Li, N. Wu, Y. Liu, Y.-Y. Hu, and J. Goodenough, High-performance all-solid-state batteries enabled by salt bonding to perovskite in poly(ethylene oxide), Proc. Natl. Acad. Sci. USA 116, 18815 (2019).

[3] D. Staedler, T. Magouroux, S. Passemard, S. Schwung, M. Dubled, G. Schneiter, D. Rytz, S. Gerber, L. Bonacina, and J.-P. Wolf, Deep UV generation and direct DNA photo-interaction by harmonic nanoparticles in labelled samples, Nanoscale 6, 2929 (2014).

[4] J. A. Alonso, M. J. Martínez-Lope, M. T. Casais, J. L. GarcíaMuñoz, M. T. Fernández-Díaz, and M. A. G. Aranda, Hightemperature structural evolution of $\mathrm{RNiO}_{3}(\mathrm{R}=\mathrm{Ho}, \mathrm{Y}, \mathrm{Er}, \mathrm{Lu})$ perovskites: Charge disproportionation and electronic localization, Phys. Rev. B 64, 094102 (2001).

[5] M. Medarde, C. Dallera, M. Grioni, B. Delley, F. Vernay, J. Mesot, M. Sikora, J. A. Alonso, and M. J. Martínez-Lope, Charge disproportionation in $\mathrm{RNiO}_{3}$ perovskites $(\mathrm{R}=$ rare earth) from high-resolution x-ray absorption spectroscopy, Phys. Rev. B 80, 245105 (2009).

[6] S. Johnston, A. Mukherjee, I. Elfimov, M. Berciu, and G. A. Sawatzky, Charge Disproportionation without Charge Transfer in the Rare-Earth-Element Nickelates as a Possible Mechanism for the Metal-Insulator Transition, Phys. Rev. Lett. 112, 106404 (2014).
[7] R. J. Green, M. W. Haverkort, and G. A. Sawatzky, Bond disproportionation and dynamical charge fluctuations in the perovskite rare-earth nickelates, Phys. Rev. B 94, 195127 (2016).

[8] D. J. Gawryluk, Y. M. Klein, T. Shang, D. Sheptyakov, L. Keller, N. Casati, P. Lacorre, M. T. Fernández-Díaz, J. Rodríguez-Carvajal, and M. Medarde, Distortion mode anomalies in bulk $\mathrm{PrNiO}_{3}$ : Illustrating the potential of symmetryadapted distortion mode analysis for the study of phase transitions, Phys. Rev. B 100, 205137 (2019).

[9] R. Rana, P. Pandey, V. E. Phanindra, S. S. Prabhu, and D. S. Rana, Terahertz spectroscopic evidence of low-energy excitations in $\mathrm{NdNiO}_{3}$, Phys. Rev. B 97, 045123 (2018).

[10] P. King, H. Wei, Y. Nie, M. Uchida, C. Adamo, S. Zhu, X. He, I. Bozovic, D. Schlom, and M. Shen, Atomic-scale control of competing electronic phases in ultrathin $\mathrm{LaNiO}_{3}$, Nat. Nanotechnol. 9, 443 (2014).

[11] R. S. Dhaka, T. Das, N. C. Plumb, Z. Ristic, W. Kong, C. E. Matt, N. Xu, K. Dolui, E. Razzoli, M. Medarde, L. Patthey, M. Shi, M. Radovic, and J. Mesot, Tuning the metal-insulator transition in $\mathrm{NdNiO}_{3}$ heterostructures via Fermi surface instability and spin fluctuations, Phys. Rev. B 92, 035127 (2015).

[12] A. Hampel, P. Liu, C. Franchini, and C. Ederer, Energetics of the coupled electronic-structural transition in the rare-earth nickelates, npj Quantum Mater. 4, 5 (2019).

[13] A. B. Georgescu, O. E. Peil, A. S. Disa, A. Georges, and A. J. Millis, Disentangling lattice and electronic contributions to the metal-insulator transition from bulk vs. layer confined $\mathrm{NdNiO}_{3}$, Proc. Natl. Acad. Sci. USA 116, 14434 (2019). 
[14] J. Liu, E. Jia, L. Wang, K. Stoerzinger, H. Zhou, C. S. Tang, X. Yin, X. He, E. Bousquet, M. Bowden, A. Wee, S. Chambers, and Y. Du, Tuning the electronic structure of $\mathrm{LaNiO}_{3}$ through alloying with strontium to enhance oxygen evolution activity, Adv. Sci. 6, 1901073 (2019).

[15] J. L. Garcia-Munoz, J. Rodriguez-Carvajal, and P. Lacorre, Neutron-diffraction study of the magnetic ordering in the insulating regime of the perovskites $\mathrm{RNiO}_{3}(\mathrm{R}=\mathrm{Pr}$ and $\mathrm{Nd})$, Phys. Rev. B 50, 978 (1994).

[16] J. Rodríguez-Carvajal, S. Rosenkranz, M. Medarde, P. Lacorre, M. T. Fernandez-Díaz, F. Fauth, and V. Trounov, Neutrondiffraction study of the magnetic and orbital ordering in ${ }^{154} \mathrm{SmNiO}_{3}$ and ${ }^{153} \mathrm{EuNiO}_{3}$, Phys. Rev. B 57, 456 (1998).

[17] M. T. Fernández-Díaz, J. A. Alonso, M. J. Martínez-Lope, M. T. Casais, and J. L. García-Muñoz, Magnetic structure of the $\mathrm{HoNiO}_{3}$ perovskite, Phys. Rev. B 64, 144417 (2001).

[18] A. Munoz, J. Alonso, M. Martinez-Lope, and M. FernandezDiaz, On the magnetic structure of $\mathrm{DyNiO}_{3}$, J. Solid State Chem. 182, 1982 (2009).

[19] I. Buitrago and C. Ventura, Magnetic excitations of perovskite rare-earth nickelates: $\mathrm{RNiO}_{3}$, J. Magn. Magn. Mater. 394, 148 (2015).

[20] J. Ruppen, J. Teyssier, I. Ardizzone, O. E. Peil, S. Catalano, M. Gibert, J.-M. Triscone, A. Georges, and D. van der Marel, Impact of antiferromagnetism on the optical properties of rareearth nickelates, Phys. Rev. B 96, 045120 (2017).

[21] A. Hampel and C. Ederer, Interplay between breathing mode distortion and magnetic order in rare-earth nickelates $\mathrm{RNiO}_{3}$ within DFT+U, Phys. Rev. B 96, 165130 (2017).

[22] S. Fomichev, G. Khaliullin, and M. Berciu, Effect of electronlattice coupling on charge and magnetic order in rare-earth nickelates, Phys. Rev. B 101, 024402 (2020).

[23] T. Mizokawa, D. I. Khomskii, and G. A. Sawatzky, Spin and charge ordering in self-doped Mott insulators, Phys. Rev. B 61, 11263 (2000).

[24] I. I. Mazin, D. I. Khomskii, R. Lengsdorf, J. A. Alonso, W. G. Marshall, R. M. Ibberson, A. Podlesnyak, M. J. Martínez-Lope, and M. M. Abd-Elmeguid, Charge Ordering as Alternative to Jahn-Teller Distortion, Phys. Rev. Lett. 98, 176406 (2007).

[25] H. Park, A. J. Millis, and C. A. Marianetti, Site-Selective Mott Transition in Rare-Earth-Element Nickelates, Phys. Rev. Lett. 109, 156402 (2012).

[26] A. Subedi, O. E. Peil, and A. Georges, Low-energy description of the metal-insulator transition in the rare-earth nickelates, Phys. Rev. B 91, 075128 (2015).

[27] J. Ruppen, J. Teyssier, O. E. Peil, S. Catalano, M. Gibert, J. Mravlje, J.-M. Triscone, A. Georges, and D. van der Marel, Optical spectroscopy and the nature of the insulating state of rare-earth nickelates, Phys. Rev. B 92, 155145 (2015).

[28] Y. M. Klein, M. Kozlowski, A. Linden, P. Lacorre, M. Medarde, and D. J. Gawryluk, $\mathrm{RENiO}_{3}$ single crystals $(\mathrm{RE}=\mathrm{Nd}, \mathrm{Sm}$, Gd, Dy, Y, Ho, Er, Lu) grown from molten salts under 2000 bar oxygen-gas pressure, arXiv:2104.09873.

[29] N. Palina, L. Wang, S. Dash, X. Yu, M. Breese, J. Wang, and A. Rusydi, Investigation of the metal-insulator transition in $\mathrm{NdNiO}_{3}$ films by site-selective X-ray absorption spectroscopy, Nanoscale 9, 6094 (2017).

[30] K. Post, A. McLeod, M. Hepting, M. Bluschke, Y. Wang, G. Cristiani, G. Logvenov, A. Charnukha, G. Ni, P. Radhakrishnan, M. Minola, A. Pasupathy, A. Boris, E. Benckiser, K. Dahmen,
E. Carlson, B. Keimer, and D. Basov, Coexisting first- and second-order electronic phase transitions in a correlated oxide, Nat. Phys. 14, 1056 (2018).

[31] R. S. Bisht, S. Samanta, and A. K. Raychaudhuri, Phase coexistence near the metal-insulator transition in a compressively strained $\mathrm{NdNiO}_{3}$ film grown on $\mathrm{LaAlO}_{3}$ : Scanning tunneling, noise, and impedance spectroscopy studies, Phys. Rev. B 95 , 115147 (2017).

[32] V. Scagnoli, U. Staub, A. M. Mulders, M. Janousch, G. I. Meijer, G. Hammerl, J. M. Tonnerre, and N. Stojic, Role of magnetic and orbital ordering at the metal-insulator transition in $\mathrm{NdNiO}_{3}$, Phys. Rev. B 73, 100409(R) (2006).

[33] V. Scagnoli, U. Staub, Y. Bodenthin, M. García-Fernández, A. M. Mulders, G. I. Meijer, and G. Hammerl, Induced noncollinear magnetic order of $\mathrm{Nd}^{3+}$ in $\mathrm{NdNiO}_{3}$ observed by resonant soft x-ray diffraction, Phys. Rev. B 77, 115138 (2008).

[34] D. Meyers, S. Middey, M. Kareev, J. Liu, J. W. Kim, P. Shafer, P. J. Ryan, and J. Chakhalian, Charge order and antiferromagnetism in epitaxial ultrathin films of $\mathrm{EuNiO}_{3}$, Phys. Rev. B 92 235126 (2015).

[35] Y. Lu, D. Betto, K. Fürsich, H. Suzuki, H.-H. Kim, G. Cristiani, G. Logvenov, N. B. Brookes, E. Benckiser, M. W. Haverkort, G. Khaliullin, M. Le Tacon, M. Minola, and B. Keimer, Siteselective probe of magnetic excitations in rare-earth nickelates using resonant inelastic X-ray scattering, Phys. Rev. X 8, 031014 (2018).

[36] S.-W. Cheong and M. Mostovoy, Multiferroics: A magnetic twist for ferroelectricity, Nat. Mater. 6, 13 (2007).

[37] J. van den Brink and D. I. Khomskii, Multiferroicity due to charge ordering, J. Phys.: Condens. Matter 20, 434217 (2008).

[38] G. Catalan, Progress in perovskite nickelate research, Phase Transitions 81, 729 (2008).

[39] G. Giovannetti, S. Kumar, D. Khomskii, S. Picozzi, and J. van den Brink, Multiferroicity in Rare-Earth Nickelates $\mathrm{RNiO}_{3}$, Phys. Rev. Lett. 103, 156401 (2009).

[40] X. Hao, A. Stroppa, P. Barone, A. Filippetti, C. Franchini, and S. Picozzi, Structural and ferroelectric transitions in magnetic nickelate $\mathrm{PbNiO}_{3}$, New J. Phys. 16, 015030 (2013).

[41] C. Xin, Y. Wang, Y. Sui, Y. Wang, X. Wang, Y. Su, K. Zhao, and $\mathrm{X}$. Liu, Electronic, magnetic and ferroelectric properties of multiferroic $\mathrm{TlNiO}_{3}$ : A first principles study, Comput. Mater. Sci. 82, 191 (2014).

[42] D. I. Khomskii, Coupled Electricity and Magnetism in Solids: Multiferroics and Beyond (Taylor and Francis, CRC Press, Boca Raton, London, New York, 2017) Chap. 1, pp. 1-31.

[43] J. M. Perez-Mato, S. V. Gallego, L. Elcoro, E. Tasci, and M. I. Aroyo, Symmetry conditions for type II multiferroicity in commensurate magnetic structures, J. Phys.: Condens. Matter 28, 286001 (2016).

[44] C. Xin, Z. Sun, B. Yuan, Z. Wei, H. Li, Z. Hu, and G. Jin, Insight into the effect of high pressure on multiferroic properties of $\mathrm{TmNiO}_{3}$, J. Alloys Compd. 815, 152351 (2020).

[45] N. A. Spaldin and R. Ramesh, Advances in magnetoelectric multiferroics, Nat. Mater. 18, 203 (2019).

[46] C. Girardot, J. Kreisel, S. Pignard, N. Caillault, and F. Weiss, Raman scattering investigation across the magnetic and metalinsulator transition in rare earth nickelate $R \mathrm{NiO}_{3}(R=\mathrm{Sm}, \mathrm{Nd})$ thin films, Phys. Rev. B 78, 104101 (2008). 
[47] M. Zaghrioui, A. Bulou, P. Lacorre, and P. Laffez, Electron diffraction and Raman scattering evidence of a symmetry breaking at the metal-insulator transition of $\mathrm{NdNiO}_{3}$, Phys. Rev. B 64, 081102(R) (2001).

[48] The propogation vector in $P 2_{1} / n$ setting is $(1 / 2,0,1 / 2)$. In $P 2_{1} / c$ setting it is $(1 / 2,0,0)$, corresponding to doubling of the paramagnetic primitive cell along $a$.

[49] R. D. Shannon, Revised effective ionic radii and systematic studies of interatomic distances in halides and chalcogenides, Acta Crystallogr., Sect. A 32, 751 (1976).

[50] F. Serrano-Sánchez, J. L. Martínez, F. Fauth, and J. A. Alonso, On the lack of monoclinic distortion in the insulating regime of $\mathrm{EuNiO}_{3}$ and $\mathrm{GdNiO}_{3}$ perovskites by high-angular resolution synchrotron X-ray diffraction: A comparison with $\mathrm{YNiO}_{3}$, Dalton Trans. 50, 7085 (2021).

[51] I. Ardizzone, J. Teyssier, I. Crassee, A. B. Kuzmenko, D. G. Mazzone, D. J. Gawryluk, M.
Medarde, and D. van der Marel, Open Data (2021), doi:10.26037/yareta:5g6gnblfnbfc7fndlp4ijntxdy.

[52] A. B. Kuzmenko, Kramers-Kronig constrained variational analysis of optical spectra, Rev. Sci. Instrum. 76, 083108 (2005).

[53] P. Giannozzi et al., QUANTUM ESPRESSO: A modular and open-source software project for quantum simulations of materials, J. Phys.: Condens. Matter 21, 395502 (2009).

[54] J. P. Perdew, K. Burke, and M. Ernzerhof, Generalized Gradient Approximation Made Simple, Phys. Rev. Lett. 77, 3865 (1996).

[55] C. J. Bradley and A. P. Cracknell, The Mathematical Theory of Symmetry in Solids (Clarendon Press, Oxford/New York, 2010).

[56] D. B. Litvin, Magnetic Group Tables: 1-, 2- and 3-Dimensional Magnetic Subperiodic Groups and Magnetic Space Groups, Vol. 1-2 (International Union of Crystallography, Chester, England, 2013). 